\title{
Psychological Health Status and Oral Health Outcomes of Pregnant Women: Practical Implications
}

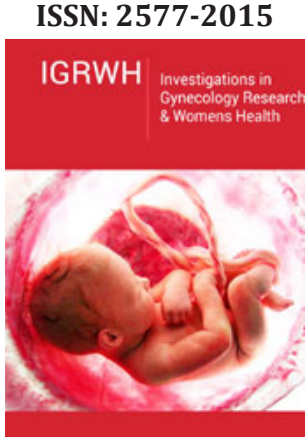

*Corresponding author: Irosha Perera, Preventive Oral Health Unit, National Dental Hospital (Teaching), Sri Lanka

Submission: 制April 26, 2019

Published: 恝May 15, 2019

Volume 2 - Issue 5

How to cite this article: Irosha P, Lushan H, Manosha P. Psychological Health Status and Oral Health Outcomes of Pregnant Women: Practical Implications. Invest Gynecol Res Women's Health.2(5). IGRWH.000549.2019.

DOI: 10.31031/IGRWH.2019.02.000549

Copyright@ Irosha Perera, This article is distributed under the terms of the Creative Commons Attribution 4.0 International License, which permits unrestricted use and redistribution provided that the original author and source are credited.

\author{
Irosha Perera' ${ }^{1 *}$, Lushan Hettiarachchige ${ }^{2}$ and Manosha Perera ${ }^{3}$ \\ ${ }^{1}$ Preventive Oral Health Unit, National Dental Hospital (Teaching), Sri Lanka \\ ${ }^{2}$ Forensic Psychiatric Unit, National Institute of Mental Health, Sri Lanka \\ ${ }^{3}$ Department of Medical Laboratory Science, Sri Lanka
}

\section{Opinion}

Pregnancy marks an important milestone in the life cycle of a woman garnered by a milieu of physiological, psychological, emotional, social and even spiritual health needs [1]. Ensuring safe motherhoods and pregnancy outcomes for a healthy mother and a baby have become priorities for health policy makers and health care delivery models both in developed and developing country scenarios. Moreover, pregnancy denotes an opportune time for intergenerational investment in health outcomes. It is well known that physiological changes that are taking place during pregnancy could give rise to demanding psychological health concerns among pregnant women compounded by their social determinates such as i.e. level of education, marital status and satisfaction, level of income, parity, minority status, having health insurance, access to health care etc. [2]. Adverse life circumstances could contribute to negative psychological status of pregnant women such as stress, depression, anxiety and much more [3]. These could progress into sinister events such as post-partum depression and even to post-partum psychosis in the context of predisposing mental conditions [3].

There is sound evidence to substantiate that pregnancy increases the risk of common oral diseases such as dental caries due to altered dietary patterns with frequent consumption of cariogenic snacks and periodontal diseases due to hormonal changes associated with pregnancy and some difficulties in maintaining optimal oral hygiene [1]. Consequently, there could be worsening of existing oral diseases of a pregnant woman and a high oral disease burden. In general, $80-90 \%$ of pregnant women in developing countries present with untreated dental caries and periodontal disease [1]. Research evidence supports increased risk of adverse neonatal outcomes such as pre-eclampsia, low birth weight, pre-term births, intra-uterine growth retardation and pregnancy outcomes such as gestational diabetes associated with high periodontal disease burden of pregnant mothers [4]. The resemblance of oral microbiome of the pregnant women with her placental microbiome as revealed by recent metagenomic studies provides the biological plausibility of potential transmission of periodontopathic oral bacteria and their toxic metabolites to the fetus via placenta [5]. Furthermore, untreated dental caries of a pregnant woman gives rise to high salivary bacterial counts which increases the risk of cariogenic bacterial transmission to the baby thus increasing the burden of early childhood dental caries [6]. Against this backdrop, oral health is an important public health concern among pregnant women there by encouraging them to use preventive and curative oral health care services. Recent studies reported that there was 2-3-fold increased risk of tooth loss and non-utilization of oral health care services among pregnant mothers with life time diagnosis of anxiety. Pregnancy exposes a woman to a higher level of anxiety and depression compounded by her negative social determinants and life circumstances such as social disadvantage and lack of social support [7]. For example, recent meta-analysis revealed that the point prevalence estimates of prenatal depression 
were ranging from $8.5 \%-11 \%$ in different trimesters [8]. Therefore, mental health concerns and psychological well-being of pregnant mothers should be given a high priority. Poor oral health status and depression could coexist among pregnant mothers who belong to disadvantaged social backgrounds. Stress, anxiety and depression could increase the risk of poor oral health status by directly impacting on neuro-endocrinal and immune mechanisms as well as by stimulating risk habits which are not conducive for oral health such as cariogenic dietary habits, less optimal brushing habits etc. $[7,9]$. Hence, in the light of such evidence, it becomes important to consider the bi-directional relationship of psychological status and oral health status of pregnant mothers. Against this backdrop, screening for antenatal anxiety and depression as well as for oral diseases during pregnancy should be in place and oral health promotion and mental health promotion of pregnant women both in developed and developing country contexts especially targeted to women with social disadvantage become important. Primary Health Care Workers such as Public Health Midwives in Sri Lanka are providing field-based ante-natal and post-natal care for pregnant women with regard to mental health promotion and necessary referrals as well as for referral for oral health care.

\section{References}

1. Karunachandra NN, Perera IR, Fernando G (2012) Oral health status during pregnancy: Rural-urban comparisons of oral disease burden among ante-natal women in Sri lanka. Rural Remote Health 12: 1902.
2. Omidvar S, Faramarzi M, Tilak SH, Amn FN (2018) Associates of psychosocial factors with pregnancy healthy lifestyles. PLoS One 13(1): e0191723.

3. Alessandra B, Susan C, Pawlby S, Pariante CM (2016) Identifying the women at risk of antenatal anxiety and depression: A systematic review. J Affect Disord 191: 62-77.

4. Silveira ML, Whitcomb BW, Pekow PS, Carbone ET, Taber LC (2016) Anxiety, depression and oral health among US pregnant women: 2010 behavioral risk factor surveillance system. J Public Health Dent 76(1): 56-64.

5. Perera IR, Perera ML, Ratnasekera N (2018) The pregnancy microbiome: The link between maternal periodontitis and adverse pregnancy outcomes: Opinion. Invest Gynecol Res Women's Health 2(1): 1-2.

6. Berkowitz RJ (2003) Acquisition and transmission of mutans streptococci. J Calif Dent Assoc 31(2): 135-138.

7. Goodman JH, Chenausky KL, Freeman MP (2014) Anxiety disorders during pregnancy: a systematic review. J Clin Psychiatry 75(10): e115384.

8. Gavin NI, Gaynes BN, Lohr KN, Brody SM, Gartlehner G, et al. (2005) Perinatal depression: a systematic review of prevalence and incidence. Obstet Gynecol 106(5 Pt 1): 1071-1083.

9. Goodman JH, Chenausky KL, Freeman MP (2014) Anxiety disorders during pregnancy: a systematic review. J Clin Psychiatry 75(10): e115384. 